\title{
THE MAXIMAL CLASS WITH RESPECT TO MAXIMUMS FOR THE FAMILY OF UPPER SEMICONTINUOUS STRONG ŚWIA̧TKOWSKI FUNCTIONS
}

\author{
PAUlina SzCZUKA \\ (Communicated by Ján Borsik)
}

\begin{abstract}
The main goal of this paper is to characterize the maximal class with respect to maximums for the family of upper semicontinuous strong Świątkowski functions.

$$
\begin{gathered}
\text { (C) } 2014 \\
\text { Mathematical Institute }
\end{gathered}
$$$$
\text { Slovak Academy of Sciences }
$$

\section{Introduction}

We use mostly standard terminology and notation. The letters $\mathbb{R}$ and $\mathbb{N}$ denote the real line and the set of positive integers, respectively. The symbol $\mathrm{I}(a, b)$ denotes the open interval with endpoints $a$ and $b$. For each $A \subset \mathbb{R}$ we use the symbols int $A, \mathrm{cl} A, \mathrm{bd} A$, and $|A|$ to denote the interior, the closure, the boundary, and the outer Lebesgue measure of $A$, respectively. The Euclidean metric in $\mathbb{R}$ will be denoted by dist.

Let $I$ be a nondegenerate interval and $f: I \rightarrow \mathbb{R}$. We say that $f$ is a Darboux function $(f \in \mathscr{D})$, if it maps connected sets onto connected sets. The symbols $\mathscr{C}(f)$ and $\mathscr{C}^{-}(f)$ will stand for the set of points of continuity and left-hand continuity of $f$, respectively. We say that $f$ is a strong Swiatkowski function [4] $\left(f \in \hat{\mathcal{S}}_{s}\right)$, if whenever $\alpha, \beta \in I, \alpha<\beta$, and $y \in \mathrm{I}(f(\alpha), f(\beta))$, there is an $x_{0} \in(\alpha, \beta) \cap \mathscr{C}(f)$ such that $f\left(x_{0}\right)=y$. The symbols $\mathscr{C}$ and usc denote families

2010 Mathematics Subject Classification: Primary 26A21, 54C30; Secondary 26A15, $54 \mathrm{C} 08$.

Keywords: Darboux function, strong Świątkowski function, upper semicontinuous function, maximum of functions.

This work was supported by Kazimierz Wielki University. 
of all continuous and upper semicontinuous functions, respectively. The function $f$ is upper semicontinuous strong Światkowski $\left(f \in \dot{\mathcal{S}}_{\text {susc }}\right)$, if it is both upper semicontinuous and strong Świątkowski. (Clearly $\mathcal{S}_{\text {susc }} \subset \mathcal{S}_{s} \subset \mathscr{D}$ and both inclusions are proper.) We say that $f \in \mathscr{C}$ if and only if $f[I]$ is a singleton. The symbol $[f=a]$ stands for the set $\{x \in I: f(x)=a\}$. Similarly we define the symbols $[f<a],[f \geq a]$, etc.

Let $f: \mathbb{R} \rightarrow \mathbb{R}$. If $A \subset \mathbb{R}$ and $x$ is a limit point of $A$, then let

$$
\varlimsup
$$

Similarly we define $\overline{\lim }\left(f, A, x^{-}\right), \underline{\lim }\left(f, A, x^{+}\right)$, etc. Moreover we write $\overline{\lim }\left(f, x^{-}\right)$ instead of $\overline{\lim }\left(f, \mathbb{R}, x^{-}\right)$, etc. If $\mathscr{L}$ is a family of real functions, then we define the maximal class with respect to maximums for $\mathscr{L}$ as follows:

$$
\mathcal{M}_{\max }(\mathscr{L})=\{f: \underset{g \in \mathscr{L}}{\forall} \max \{f, g\} \in \mathscr{L}\} .
$$

It is known that $\mathcal{M}_{\max }($ usc) $=$ usc (see e.g. [2]). In 1971 Farková characterized the maximal class with respect to maximums for the family of Darboux functions, which is equal to the family of Darboux upper semicontinuous functions [1]. In 2003 I proved that $\mathcal{M}_{\max }\left(\mathcal{S}_{s}\right)=\mathscr{C}([5])$. In this paper we characterize the maximal class with respect to maximums for the family of upper semicontinuous strong Świątkowski functions. It turns out that $\mathcal{M}_{\max }\left(\mathcal{S}_{\text {susc }}\right)$ consist of upper semicontinuous strong Świątkowski functions which satisfied some special conditions. (Theorems 2.4 and 2.5). In particular the maximum of a continuous function and an upper semicontinuous strong Świątkowski function is upper semicontinuous strong Świątkowski (Corollary 2.6).

\section{Main results}

Lemma 2.1 can be easily proved using [3: Theorem 12].

Lemma 2.1. Let $I \subset \mathbb{R}$ be an interval, the function $g: I \rightarrow \mathbb{R}$, and $x \in I$. If $g\left\lceil I \cap(-\infty, x] \in \mathcal{S}_{s}, g\left\lceil I \cap(x, \infty) \in \mathcal{S}_{s}\right.\right.$, and $g(x) \in g[[x, t] \cap \mathscr{C}(g)]$ for each $t \in(x, \sup I)$, then $g \in \mathcal{S}_{s}$.

The proof of Lemma 2.2 we can find in [6: Lemma 3.4].

Lemma 2.2. Assume that $F \subset C$ are closed and $\mathcal{J}$ is a family of components of $\mathbb{R} \backslash C$ such that $C \subset \operatorname{cl} \cup \mathcal{J}$. There is a family $\mathcal{J}^{\prime} \subset \mathcal{J}$ such that

a) for each $J \in \mathcal{J}$, if $F \cap \mathrm{bd} J \neq \emptyset$, then $J \in \mathcal{J}^{\prime}$,

b) for each $c \in F$, if $c$ is a right-hand (left-hand) limit point of $C$, then $c$ is a right-hand (respectively left-hand) limit point of the union $\bigcup \mathcal{J}^{\prime}$,

c) $\operatorname{cl} \bigcup \mathcal{J}^{\prime} \subset F \cup \bigcup_{J \in \mathcal{J}^{\prime}} \operatorname{cl} J$. 
Remark 2.3. Let $f: \mathbb{R} \rightarrow \mathbb{R}$. Clearly, if the function $f$ is Darboux upper semicontinuous, then $\overline{\lim }\left(f, x^{-}\right)=f(x)=\varlimsup \lim \left(f, x^{+}\right)$for each $x \in \mathbb{R}$.

Next two theorems characterize the maximal class with respect to maximums for the family of upper semicontinuous strong Świątkowski functions.

Theorem 2.4. $\mathcal{M}_{\max }\left(\mathcal{S}_{\text {susc }}\right) \subset \mathcal{S}_{\text {susc }}$.

Pr o of. First assume that $f \notin \mathcal{S}_{s}$. Then there are $\alpha<\beta$ and $y \in \mathrm{I}(f(\alpha), f(\beta))$ such that $f(x) \neq y$ for each $x \in(\alpha, \beta) \cap \mathscr{C}(f)$. Put $g=\min \{f(\alpha), f(\beta)\}$ and $h=\max \{f, g\}$. Then clearly $g \in \mathscr{C} \subset \mathcal{S}_{\text {susc }}$. Since $y \in \mathrm{I}(h(\alpha), h(\beta))$ and $h(x) \neq y$ for each $x \in(\alpha, \beta) \cap \mathscr{C}(h)$, we have $h \notin \mathcal{S}_{s}$. So, $h \notin \dot{\mathcal{S}}_{\text {susc }}$, whence $f \notin \mathcal{M}_{\text {max }}\left(\mathcal{S}_{\text {susc }}\right)$.

Now assume that $f \notin$ usc. Then e.g., $f\left(x_{0}\right)<\overline{\lim }\left(f, x_{0}^{+}\right)$for some $x_{0} \in \mathbb{R}$. (The other case is analogous.) Put $g=f\left(x_{0}\right)$ and $h=\max \{f, g\}$. Then clearly $g \in \mathscr{C} \subset \mathcal{S}_{\text {susc }}$, and since

$$
h\left(x_{0}\right)=g\left(x_{0}\right)=f\left(x_{0}\right)<\varlimsup \lim \left(f, x_{0}^{+}\right)=\varlimsup \lim \left(h, x_{0}^{+}\right),
$$

$h \notin$ usc. So, $h \notin \dot{\mathcal{S}}_{\text {susc }}$, whence $f \notin \mathcal{M}_{\text {max }}\left(\dot{\mathcal{S}}_{\text {susc }}\right)$.

TheOREM 2.5. Assume that $f \in \mathcal{S}_{\text {susc }}$. The following conditions are equivalent:

a) there is a function $g \in \mathcal{S}_{\text {susc }}$ such that $\max \{f, g\} \notin \mathcal{S}_{s}$,

b) there are: real numbers $a<b$, a nowhere dense $G_{\delta}$-set $A \subset(a, b)$, a point $x_{0} \in A$, and a subfamily $\mathcal{J}$ of the family of all components of $[a, b] \backslash \operatorname{cl} A$ such that

(i) $\operatorname{cl} A \subset \operatorname{cl} \cup \mathcal{J}$,

(ii) $\bigcup_{J \in \mathcal{J}} \operatorname{bd} J \cap(a, b) \subset A \cap \operatorname{cl}\left[f>f\left(x_{0}\right)\right]$,

(iii) $\bigcup \mathcal{J} \subset \operatorname{int}\left(\left[f<f\left(x_{0}\right)\right] \cup\left(\left[f=f\left(x_{0}\right)\right] \backslash \mathscr{C}(f)\right)\right)$,

(iv) $\underline{\lim }(f, \bigcup \mathcal{J}, x)<f\left(x_{0}\right)$ for each $x \in A$.

Proof. Assume that $f \in \mathcal{S}_{\text {susc }}$.

NeCEssity.

Let $g \in \mathcal{S}_{\text {susc }}$ and $h=\max \{f, g\} \notin \mathcal{S}_{s}$. Then there are $a<b$ and $y \in \mathrm{I}(h(a), h(b))$ such that

$$
h(x) \neq y \quad \text { for each } \quad x \in(a, b) \cap \mathscr{C}(h) .
$$

Since the maximum of two upper semicontinuous functions is upper semicontinuous (see e.g. 2, p. 83]), $h \in$ usc.

Define

$$
G_{1}=\operatorname{int}[h \leq y] \quad \text { and } \quad G_{2}=\operatorname{int}[h \geq y] .
$$

Clearly sets $G_{1}$ and $G_{2}$ are nonempty, open and disjoint in $[a, b]$. Assume that $\mathcal{I}_{1}$ and $\mathcal{I}_{2}$ are families of all components of $G_{1} \cap(a, b)$ and $G_{2} \cap(a, b)$, respectively. 
Moreover let $\mathcal{I}=\mathcal{I}_{1} \cup \mathcal{I}_{2}$ and $C=[a, b] \backslash\left(G_{1} \cup G_{2}\right)$. Obviously the set $C$ is closed and nonempty, and since $h \in$ usc,

$$
C \cap(a, b) \subset[h \geq y] .
$$

We will show that $C$ is nowhere dense.

Suppose, on the contrary, that $\operatorname{int} \operatorname{cl} C \neq \emptyset$. Then there is an open interval $I \subset C$. Using condition (11) we obtain that $h(x) \neq y$ for some $x \in I$. If $h(x)>y$, then $f(x)>y$ or $g(x)>y$. Without loss of generality we can assume that $f(x)>y$. Since $f \in \dot{\mathcal{S}}_{s}$, there is a $t \in I \cap \mathscr{C}(f)$ such that $f(t)>y$. So, $(t-\delta, t+\delta) \cap I \subset[f>y]$ for some $\delta>0$, whence $(t-\delta, t+\delta) \cap I \subset[h>y]$. It proves that $(t-\delta, t+\delta) \cap I \subset G_{2}$, an impossibility. If $h(x)<y$, then $f(x)<y$ and $g(x)<y$. Since $f, g \in$ usc, there is a $\delta>0$ such that $(x-\delta, x+\delta) \cap I \subset$ $[f<y] \cap[g<y]$. Hence $(x-\delta, x+\delta) \cap I \subset[h<y]$, which proves that $(x-\delta, x+\delta) \cap I \subset G_{1}$, a contradiction. So, the set $C$ is nowhere dense.

Now we will show some properties of the set $\bigcup_{I \in \mathcal{I}_{1}}$ bd $I$. First observe that

$$
\bigcup_{I \in \mathcal{I}_{1}} \operatorname{bd} I \cap(a, b) \subset \operatorname{cl}[h>y] .
$$

Moreover,

$$
\bigcup_{I \in \mathcal{I}_{1}} \operatorname{bd} I \cap(a, b) \subset \operatorname{cl}[f>y] .
$$

Indeed, let $x \in(a, b)$ and $x=\sup I$ for some $I \in \mathcal{I}_{1}$. (The case $x=\inf I$ for some $I \in \mathcal{I}_{1}$ is analogous.) If there was a $\delta>0$ such that $f(t) \leq y$ for each $t \in(x, x+\delta)$, then from (3) and since $h=\max \{f, g\}$, there was a $t_{\delta} \in(x, x+\delta)$ with $g\left(t_{\delta}\right)>y$. But $I \subset[h \leq y]$, whence, by (11), we would have $g(z)<y$ for some $z \in I$. Since $g \in \dot{\mathcal{S}}_{s}$, there was a $t_{0} \in\left(z, t_{\delta}\right) \cap \mathscr{C}(g)$ such that $g\left(t_{0}\right)=y$. Moreover $\left(z, t_{\delta}\right) \subset[f \leq y]$. Using $h=\max \{f, g\}$ one more time, we would obtain $g\left(t_{0}\right)=h\left(t_{0}\right)=y$ and $t_{0} \in \mathscr{C}(h)$, which contradicts (1).

In the same way we can prove that

$$
\bigcup_{I \in \mathcal{I}_{1}} \operatorname{bd} I \cap(a, b) \subset \operatorname{cl}[g>y] .
$$

Finally we will show that

$$
h(x)=f(x)=g(x)=y \quad \text { for each } \quad x \in \bigcup_{I \in \mathcal{I}_{1}} \text { bd } I \cap(a, b) .
$$

Let $x \in(a, b)$ and $x=\sup I$ for some $I \in \mathcal{I}_{1}$. (The case $x=\inf I$ for some $I \in \mathcal{I}_{1}$ is analogous.) By condition (2), $h(x) \geq y$. If $h(x)>y$, then $f(x)>y$ or $g(x)>y$. But $I \subset[h \leq y]$, whence $I \subset[f \leq y] \cap[g \leq y]$. It contradicts $f, g \in \dot{\mathcal{S}}_{s} \subset \mathscr{D}$ and consequently $h(x)=y$. If $g(x)<y$, then since $g \in$ usc, there was a $\delta>0$ such that $(x-\delta, x+\delta) \subset[g<y]$, which contradicts (5). Thus $y=h(x) \geq g(x) \geq y$, whence $g(x)=y$. In the similar way we can show that $f(x)=y$. So, condition (6) is fulfilled. 
Next we claim that

$$
I \subset \operatorname{int}([f<y] \cup([f=y] \backslash \mathscr{C}(f))) \quad \text { for each } \quad I \in \mathcal{I}_{1} .
$$

Indeed, fix an $I \in \mathcal{I}_{1}$. Since $h=\max \{f, g\}$, we have $[h<y] \subset[f<y]$, and by (11),

$$
[h=y] \cap(a, b) \subset[f<y] \cup([f=y] \backslash \mathscr{C}(f)) .
$$

Hence, using definition of $\mathcal{I}_{1}$, we obtain

$$
I \subset \operatorname{int}[h \leq y] \cap(a, b) \subset \operatorname{int}([f<y] \cup([f=y] \backslash \mathscr{C}(f))),
$$

as claimed.

Now we will prove that all our requirements are fulfilled. For each $n \in \mathbb{N}$ define

$$
F_{n}=\operatorname{cl}\left(\left[h>y+\frac{1}{n}\right] \cap C\right) .
$$

Let $F=\bigcup_{n \in \mathbb{N}} F_{n} \cup\{a, b\}$. Then $F$ is an $F_{\sigma}$-set. Define $A=C \backslash F$. Clearly $A$ is a nowhere dense $G_{\delta}$-set and $A \subset(a, b)$. Now we will show that

$$
C \subset \operatorname{cl} \cup \mathcal{I}_{1} \cup\{a, b\} .
$$

Let $x \in C \backslash\{a, b\}$. If (8) was not fulfilled, then there was a $\delta>0$ such that $(x-\delta, x+\delta) \subset[a, b]$ and $(x-\delta, x+\delta) \cap \bigcup \mathcal{I}_{1}=\emptyset$. But then $(x-\delta, x+\delta) \subset C \cup \bigcup \mathcal{I}_{2}$, and by (2), we would have $(x-\delta, x+\delta) \subset[h \geq y]$. Hence $(x-\delta, x+\delta) \subset G_{2}$, a contradiction.

Moreover

$$
\bigcup_{I \in \mathcal{I}_{1}} \operatorname{bd} I \subset A \cup\{a, b\} .
$$

Indeed, let $x \in \operatorname{bd} I \backslash\{a, b\}$ for some $I \in \mathcal{I}_{1}$. Hence obviously $x \in C$. If $x \in F$, then $x \in F_{n}$ for some $n \in \mathbb{N}$. Since $h \in$ usc, we would have $h(x) \geq y+\frac{1}{n}$, which contradicts (6). Therefore $x \in A$, whence condition (9) holds.

Now observe that conditions (8) and (9) imply cl $A \cup\{a, b\}=C \cup\{a, b\}$. So, we can assume that $\mathcal{J}=\mathcal{I}_{1}$. Then $\mathcal{J}$ is a subfamily of the family of all components of $[a, b] \backslash \operatorname{cl} A$. Choose an $I_{0} \in \mathcal{J}$ such that $\sup I_{0} \cap\{a, b\} \neq \emptyset$ and let $x_{0}=\sup I_{0}$. Clearly $x_{0} \in A$. It remains to prove that conditions (i)-(iv) are fulfilled.

Condition (i) follows from (8) . (Recall that $A \subset C \backslash\{a, b\}$.) Using (9) we obtain that

$$
\bigcup_{J \in \mathcal{J}} \operatorname{bd} J \cap(a, b) \subset A .
$$

Since $x_{0} \in \bigcup_{J \in \mathcal{J}}$ bd $J \cap(a, b)$, by (6),$f\left(x_{0}\right)=y$. Therefore, by (4),

$$
\bigcup_{J \in \mathcal{J}} \operatorname{bd} J \cap(a, b) \subset \operatorname{cl}\left[f>f\left(x_{0}\right)\right] .
$$

So, condition (ii) is fulfilled. Condition (iii) holds directly from (7). Finally, fix an $x \in A$. Observe that, by (2), $h(x) \geq y$. But if $h(x)>y$, then $x \in F$, 
a contradiction. Hence $h(x)=y$. Taking into account that $h \in$ usc, $f, g \in \mathcal{S}_{s}$, and $h=\max \{f, g\}$, we conclude that $\overline{\lim }\left(h, x^{-}\right)=\overline{\lim }\left(h, x^{+}\right)=y$. Moreover $C \cup$ $\bigcup \mathcal{I}_{2} \subset[h \geq y]$. So, if $\underline{\lim }\left(h, \bigcup \mathcal{I}_{1}, x^{-}\right)=\underline{\lim }\left(h, \bigcup \mathcal{I}_{1}, x^{+}\right)=y$, then $x \in \mathscr{C}(h)$, which contradicts (11). Therefore $\underline{\lim }\left(h, \bigcup \mathcal{I}_{1}, x^{-}\right)<y$ or $\underline{\lim }\left(h, \bigcup \mathcal{I}_{1}, x^{+}\right)<y$, whence by (6),

$$
\underline{\lim }(f, \bigcup \mathcal{J}, x)<y=f\left(x_{0}\right) .
$$

This completes first part of the proof.

SUFFICIENCY.

Now assume that there are real numbers $a<b$, a nowhere dense $G_{\delta}$-set $A \subset(a, b)$, a point $x_{0} \in A$, and a subfamily $\mathcal{J}$ of the family of all components of $[a, b] \backslash \mathrm{cl} A$ such that conditions (i)-(iv) are fulfilled. First observe that using assumptions (ii), (iii), and the fact that $f \in \mathcal{S}_{\text {susc }}$, we have

$$
f(x)=f\left(x_{0}\right) \quad \text { for each } \quad x \in \bigcup_{J \in \mathcal{J}} \operatorname{bd} J \cap(a, b) .
$$

Since $\operatorname{cl} A$ is nowhere dense we can write $\operatorname{cl} A$ as the disjoint union $\operatorname{cl} A=C \cup P$, where $P$ is countable and $C$ is perfect. We consider two cases.

Case I. $P \neq \emptyset$.

Then, by assumption (ii), there is an isolated in $A$ point $z_{0} \in P \cap(a, b) \cap \bigcup_{J \in \mathcal{J}}$ bd $J$.

Let $z_{0}=\sup J$ for some $J \in \mathcal{J}$. (If $z_{0}=\inf J$ for some $J \in \mathcal{J}$ we proceed analogously.) Then, by (10), $f\left(z_{0}\right)=f\left(x_{0}\right)$. This fact and assumption (iv) imply that $z_{0} \notin \mathscr{C}^{-}(f)$. Using assumption (iii) we obtain that

$$
f(x)<f\left(x_{0}\right) \quad \text { for each } \quad x \in J \cap \mathscr{C}(f) .
$$

Moreover, by assumption (ii) and since $f \in \mathcal{S}_{s}$, there is a sequence $\left(x_{n}\right) \subset \mathscr{C}(f)$ such that $x_{n} \rightarrow z_{0}^{+}$and $f\left(x_{n}\right)>f\left(x_{0}\right)$ for each $n \in \mathbb{N}$. Hence, for each $n \in \mathbb{N}$, there is a $\delta_{n}>0$ such that $f(x)>f\left(x_{0}\right)$ for every $x \in\left(x_{n}-\delta_{n}, x_{n}+\delta_{n}\right)$. Without loss of generality we can assume that $x_{n+1}+\delta_{n+1}<x_{n}-\delta_{n}$ for each $n \in \mathbb{N}$. Define the function $g: \mathbb{R} \rightarrow \mathbb{R}$ as follows:

$$
g(x)= \begin{cases}f(x) & \text { if } x \in\left(-\infty, z_{0}\right] \\ f\left(x_{0}\right) & \text { if } x \in\left\{x_{n}: n \in \mathbb{N}\right\} \cup\left[x_{1}, \infty\right) \\ f\left(x_{n+1}\right) & \text { if } x \in \bigcup_{n=1}^{\infty}\left[x_{n+1}+\delta_{n+1}, x_{n}-\delta_{n}\right] \\ \text { linear } & \text { in each interval }\left[x_{n+1}, x_{n+1}+\delta_{n+1}\right] \text { and }\left[x_{n}-\delta_{n}, x_{n}\right], \\ & n \in \mathbb{N} .\end{cases}
$$

Observe that $g \uparrow\left(-\infty, z_{0}\right]=f \uparrow\left(-\infty, z_{0}\right] \in \mathcal{S}_{\text {susc }}$ and $g \uparrow\left[z_{0}, \infty\right) \in \mathscr{C}$. So, clearly $g \in$ usc. Moreover, $g\left(z_{0}\right)=g\left[\left(x_{n}\right)\right]$ and $\left(x_{n}\right) \subset \mathscr{C}(g)$. Thus, by Lemma 2.1. $g \in \dot{\mathcal{S}}_{s}$. Now we will show that $h=\max \{f, g\} \notin \dot{\mathcal{S}}_{s}$. 
Take an $\alpha \in J \cap \mathscr{C}(f)$ and let $\beta=x_{1}-\delta_{1}$. Notice that, by (11), for each $x \in\left[\alpha, z_{0}\right) \cap \mathscr{C}(f)$

$$
h(x)=g(x)=f(x)<f\left(x_{0}\right) .
$$

Now fix an $x \in\left(z_{0}, \beta\right]$. Observe that $h(x)>f\left(x_{0}\right)$. Indeed,

- if $x \in\left(x_{n}-\delta_{n}, x_{n}+\delta_{n}\right)$ for some $n \in \mathbb{N}$, then $h(x) \geq f(x)>f\left(x_{0}\right)$, and

- if $x \in\left[x_{n+1}+\delta_{n+1}, x_{n}-\delta_{n}\right]$ for some $n \in \mathbb{N}$, then $h(x) \geq g(x)>f\left(x_{0}\right)$.

Hence in particular $f\left(x_{0}\right) \in(h(\alpha), h(\beta))$. Moreover, since $z_{0} \notin \mathscr{C}^{-}(f)$ and $f=g$ on $\left(-\infty, z_{0}\right]$, we have $z_{0} \notin \mathscr{C}(h)$. Therefore $h(x) \neq f\left(x_{0}\right)$ for each $x \in(\alpha, \beta) \cap \mathscr{C}(h)$. Consequently $h=\max \{f, g\} \notin \dot{\mathcal{S}}_{s}$.

Case II. $P=\emptyset$.

Then $C \neq \emptyset$ and $C=\operatorname{cl} A$. (Recall that $C$ is perfect.) Define

$$
c=\inf \{x \in[a, b]: x \in C\} \quad \text { and } d=\sup \{x \in[a, b]: x \in C\} .
$$

Observe that $c, d \in C$. Let $\mathcal{I}$ be the family of all components of $[c, d] \backslash C$. Define

$$
\mathcal{I}^{\prime}=\left\{I \in \mathcal{I}: I \cap\left[f>f\left(x_{0}\right)\right] \neq \emptyset\right\} .
$$

By (10) and assumption (ii), $\mathcal{I}^{\prime} \neq \emptyset$. Taking into account that the set $C$ is perfect and using assumptions (i) and (ii), we obtain that

$$
C=\operatorname{cl} A \subset \operatorname{cl} \bigcup_{J \in \mathcal{J}} \operatorname{bd} J \subset \operatorname{cl}\left[f>f\left(x_{0}\right)\right] .
$$

Since $f \in \mathcal{S}_{\text {susc }}$, we have $C \subset \mathrm{cl} \bigcup \mathcal{I}^{\prime}$. Now define

$$
A_{1}=A \cap C \backslash \bigcup_{I \in \mathcal{I}^{\prime}} \operatorname{bd} I .
$$

Since $A$ is a $G_{\delta}$-set, $A_{1}$ is a $G_{\delta}$-set, too. Then $C \backslash A_{1}$ is an $F_{\sigma}$-set, whence there is a sequence $\left(F_{n}\right)$ consisting of closed sets such that

$$
C \backslash A_{1}=\bigcup_{n \in \mathbb{N}} F_{n} .
$$

Define $F_{0}^{\prime}=\emptyset$. For each $n \in \mathbb{N}$, use Lemma 2.2 to construct a sequence of sets $\left(F_{n}^{\prime}\right)$ and a sequence of families of intervals $\left(\mathcal{I}_{n}^{\prime}\right)$ such that

$$
\begin{gathered}
\mathcal{I}_{n}^{\prime} \subset \mathcal{I}^{\prime}, \\
F_{n}^{\prime}=F_{n} \cup \bigcup_{k<n}\left(F_{k}^{\prime} \cup \bigcup_{I \in \mathcal{I}_{k}^{\prime}} \mathrm{bd} I\right)
\end{gathered}
$$

for each $I \in \mathcal{I}^{\prime}$, if $F_{n}^{\prime} \cap$ bd $I \neq \emptyset$, then $I \in \mathcal{I}_{n}^{\prime}$,

for each $c \in F_{n}^{\prime}$, if $c$ is a right-hand (left-hand) limit point of $C$, then $c$ is a right-hand (left-hand) limit point of the union $\bigcup \mathcal{I}_{n}^{\prime}$,

$$
\operatorname{cl} \cup \mathcal{I}_{n}^{\prime} \subset F_{n}^{\prime} \cup \bigcup_{I \in \mathcal{I}_{n}^{\prime}} \operatorname{cl} I .
$$


Note that, by (18), for each $k<n$, the set $F_{k}^{\prime} \cup\left\{\operatorname{bd} I: I \in \mathcal{I}_{k}^{\prime}\right\}$ is closed. So, by (15), the set $F_{n}^{\prime}$ is also closed and $F_{n}^{\prime} \subset C \backslash A_{1}$. Moreover, by (16), $\bigcup_{n \in \mathbb{N}} \mathcal{I}_{n}^{\prime}=\mathcal{I}^{\prime}$

Put

$$
n_{I}=\min \left\{n \in \mathbb{N}: I \in \mathcal{I}_{n}^{\prime}\right\}, \quad N_{x}=\min \left\{n \in \mathbb{N}: x \in F_{n}^{\prime}\right\}
$$

and

$$
n_{x}= \begin{cases}N_{x}-1 & \text { if } x \in\left\{\operatorname{bd} I: I \in \mathcal{I}^{\prime}\right\} \text { and } x \text { is a right-hand (left-hand) } \\ & \text { limit point of the union } \bigcup \mathcal{I}_{N_{x}-1}^{\prime}, \\ N_{x} & \text { otherwise. }\end{cases}
$$

Fix an $I=\left(a_{I}, b_{I}\right) \in \mathcal{I}^{\prime}$. Observe that, if $x \in \mathrm{bd} I$, then by (15), $\frac{1}{n_{I}+1} \leq \frac{1}{n_{x}}$ $\leq \frac{1}{n_{I}}$. Moreover, since $f \in \mathcal{S}_{s}$ and $I \cap\left[f>f\left(x_{0}\right)\right] \neq \emptyset$, there is a $z \in I \cap \mathscr{C}(f)$ with $f(x)>f\left(x_{0}\right)$. So, there is a $\delta>0$ such that $[z-\delta, z+\delta] \subset I$ and $f(x)>f\left(x_{0}\right)$ for each $x \in(z-\delta, z+\delta)$. Define the function $g_{I}: \operatorname{cl} I \rightarrow \mathbb{R}$ as follows:

$$
g_{I}(x)= \begin{cases}f\left(x_{0}\right) & \text { if } x=z, \\ f\left(x_{0}\right)+\frac{1}{n_{I}} & \text { if } x \in\{z-\delta, z+\delta\}, \\ f\left(x_{0}\right)+\frac{1}{n_{x}} & \text { if } x \in \operatorname{bd} I, \\ \text { linear } & \text { in intervals }\left[a_{I}, z-\delta\right],[z-\delta, z],[z, z+\delta], \text { and } \\ & {\left[z+\delta, b_{I}\right] .}\end{cases}
$$

Further assume that $\mathcal{I}_{1}=\mathcal{I} \backslash\left(\mathcal{I}^{\prime} \cup \mathcal{J}\right)$ and fix an $I=\left(a_{I}, b_{I}\right) \in \mathcal{I}_{1}$. Define the function $\varphi_{I}: \operatorname{cl} I \rightarrow \mathbb{R}$ as follows:

$$
\varphi_{I}(x)= \begin{cases}f\left(x_{0}\right) & \text { if } x \in \mathrm{bd} I \backslash \bigcup_{n \in \mathbb{N}} F_{n}^{\prime}, \\ f\left(x_{0}\right)+\frac{1}{n_{x}} & \text { if } x \in \mathrm{bd} I \cap \bigcup_{n \in \mathbb{N}} F_{n}^{\prime}, \\ f\left(x_{0}\right)+|I| & \text { if } x=\frac{a_{I}+b_{I}}{2}, \\ \text { linear } & \text { in intervals }\left[a_{I}, \frac{a_{I}+b_{I}}{2}\right] \text { and }\left[\frac{a_{I}+b_{I}}{2}, b_{I}\right] .\end{cases}
$$

Now define the function $\psi:[c, d] \rightarrow \mathbb{R}$ by the formula:

$$
\psi(x)= \begin{cases}f(x) & \text { if } x \in \operatorname{cl} I, I \in \mathcal{J} \\ g_{I}(x) & \text { if } x \in \operatorname{cl} I, I \in \mathcal{I}^{\prime}, \\ \varphi_{I}(x) & \text { if } x \in \operatorname{cl} I, I \in \mathcal{I}_{1} \\ f\left(x_{0}\right)+\frac{1}{n_{x}} & \text { if } x \in \bigcup_{n \in \mathbb{N}} F_{n}^{\prime} \backslash \bigcup_{I \in \mathcal{I}} \operatorname{cl} I, \\ f\left(x_{0}\right) & \text { if } x \in A_{1} \backslash \bigcup_{I \in \mathcal{I}} \operatorname{cl} I\end{cases}
$$


Observe that $A_{1} \subset\left[\psi=f\left(x_{0}\right)\right]$ and

$$
\left[\psi<f\left(x_{0}\right)\right] \subset \bigcup \mathcal{J} \subset\left[\psi \leq f\left(x_{0}\right)\right] .
$$

We we will show that $\psi \in \mathcal{S}_{s}$ usc. First we will prove that $\psi$ is upper semicontinuous. Clearly $\psi \uparrow \bigcup \mathcal{I} \in$ usc. So, let $x \in C$.

If $x \in A_{1}$, then $\psi(x)=f\left(x_{0}\right)$. Suppose that e.g., $\overline{\lim }\left(\psi, x^{-}\right)>f\left(x_{0}\right)$. (The other case is similar.) Without loss of generality we can assume that $x \neq \sup I$ for each $I \in \mathcal{I}$. Choose an $n_{0} \in \mathbb{N}$ such that $\overline{\lim }\left(\psi, x^{-}\right)>f\left(x_{0}\right)+\frac{1}{n_{0}-1}$. By (19) and construction of $\psi$ we obtain that

$$
x \in \operatorname{cl}\left(F_{n_{0}}^{\prime} \cup \cup \mathcal{I}_{n_{0}}^{\prime} \cup \bigcup_{I \in \mathcal{I}_{1},|I| \geq \frac{1}{n_{0}}} I\right) \cap(-\infty, x) .
$$

Since $A_{1} \subset C$ and $C$ is perfect, $x \notin \mathrm{cl} \cup I \cap(-\infty, x)$. Moreover, by (18), (15), (12), and (13),

$$
I \in \mathcal{I}_{1},|I| \geq \frac{1}{n_{0}}
$$

$$
\begin{aligned}
A_{1} \cap \operatorname{cl}\left(F_{n_{0}}^{\prime} \cup \cup \mathcal{I}_{n_{0}}^{\prime}\right) & \subset\left(A_{1} \cap F_{n_{0}}^{\prime}\right) \cup\left(A_{1} \cap \bigcup_{I \in \mathcal{I}_{n_{0}}^{\prime}} \operatorname{cl} I\right) \\
& \subset\left(A_{1} \cap \bigcup_{n \leq n_{0}} F_{n}^{\prime}\right) \cup\left(\left(C \backslash \bigcup_{I \in \mathcal{I}^{\prime}} \operatorname{bd} I\right) \cap \bigcup_{I \in \mathcal{I}^{\prime}} \operatorname{cl} I\right)=\emptyset,
\end{aligned}
$$

a contradiction. So, $\psi$ is upper semicontinuous on $A_{1}$.

If $x \notin A_{1}$, then $x \in \bigcup_{n \in \mathbb{N}} F_{n}$. Hence $x \in F_{n}^{\prime} \backslash F_{n-1}^{\prime}$ for some $n \in \mathbb{N}$. Suppose that e.g., $\overline{\lim }\left(\psi, x^{-}\right)>f\left(x_{0}\right)+\frac{1}{n}$. (The other case is similar.) If $x=\sup I$ for some $I \in \mathcal{I}$, then by (10) and construction of $\psi$ we have $\overline{\lim }\left(\psi, x^{-}\right) \leq f\left(x_{0}\right)+\frac{1}{n}$, a contradiction. So let $x \neq \sup I$ for each $I \in \mathcal{I}$. Note that $x \in \operatorname{cl}([c, x] \cap[\psi>$ $\left.\left.f\left(x_{0}\right)+\frac{1}{n}\right]\right)$, whence $x \in \operatorname{cl} \bigcup \mathcal{I}_{n-1}^{\prime}$. But by (18),$x \in \bigcup_{I \in \mathcal{I}_{n}^{\prime}} \operatorname{cl} I$. Hence there is an $I \in \mathcal{I}$ such that $x=\sup I$, which is impossible. So, $\varlimsup \lim \left(\psi, x^{-}\right) \leq f\left(x_{0}\right)+\frac{1}{n}$ $=\psi(x)$. It follows that $\psi \in$ usc.

Now we will prove that for each $n \in \mathbb{N}$ and $\delta>0$, if $x \neq c$ and $x \in F_{n}^{\prime} \backslash\{\sup I$ : $I \in \mathcal{I}\}$, then

$$
\psi[(x-\delta, x) \cap \mathscr{C}(\psi)] \supset\left[f\left(x_{0}\right), f\left(x_{0}\right)+\frac{1}{n}\right] .
$$

Let $n \in \mathbb{N}, \delta>0, x \neq c$, and $x \in F_{n}^{\prime} \backslash\{\sup I: I \in \mathcal{I}\}$. Then $x \in$ $F_{n}^{\prime} \cap \operatorname{cl}((-\infty, x) \cap C)$ and by (17), there is an $I \in \mathcal{I}_{n}^{\prime}$ with $I \subset(x-\delta, x)$. Notice that $n_{I} \leq n$. So,

$$
\psi[(x-\delta, x) \cap \mathscr{C}(\psi)] \supset \psi[I \cap \mathscr{C}(\psi)] \supset\left[f\left(x_{0}\right), f\left(x_{0}\right)+\frac{1}{n}\right] .
$$

Similarly we can prove that for each $n \in \mathbb{N}$ and $\delta>0$, if $x \neq d$ and $x \in$ $F_{n}^{\prime} \backslash\{\inf I: I \in \mathcal{I}\}$, then

$$
\psi[(x, x+\delta) \cap \mathscr{C}(\psi)] \supset\left[f\left(x_{0}\right), f\left(x_{0}\right)+\frac{1}{n}\right] .
$$


Now we will show that $\psi \in \dot{\mathcal{S}}_{s}$. Let $\alpha, \beta \in[c, d], \alpha<\beta$, and $y \in \mathrm{I}(\psi(\alpha), \psi(\beta))$. Assume that $\psi(\alpha)<\psi(\beta)$. (The other case is similar.) If $\alpha, \beta \in \operatorname{cl} I$ for some $I \in \mathcal{I}$, then since $\psi\left\lceil\operatorname{cl} I \in \dot{\mathcal{S}}_{s}\right.$, there is a $t_{0} \in(\alpha, \beta) \cap \mathscr{C}(\psi)$ with $\psi\left(t_{0}\right)=y$. So, assume that the opposite case holds. We consider two cases.

Case 1. If $y \geq f\left(x_{0}\right)$, then $\psi(\beta)>f\left(x_{0}\right)$ and $\beta \notin A_{1}$.

First assume that $\beta \notin \bigcup_{n \in \mathbb{N}} F_{n}^{\prime}$ or $\beta \in\{\sup I: I \in \mathcal{I}\}$. Then there is an $I \in \mathcal{I}$ such that $\beta \in \operatorname{cl} I$ and $\alpha \notin \operatorname{cl} I$. If $y \in \mathrm{I}(\psi(\inf I), \psi(\beta))$, then since $\psi \uparrow \mathrm{cl} I \in \mathcal{S}_{s}$, there is a $t_{0} \in(\inf I, \beta) \cap \mathscr{C}(\psi) \subset(\alpha, \beta) \cap \mathscr{C}(\psi)$ with $\psi\left(t_{0}\right)=y$. So, let $y \in\left[f\left(x_{0}\right), \psi(\inf I)\right]$.

- If $\inf I \in A_{1}$, then $\psi(\inf I)=f\left(x_{0}\right)=y$ and since $\inf I \in C \subset \operatorname{cl} \bigcup \mathcal{I}^{\prime}$, there is an $I^{\prime} \in \mathcal{I}^{\prime}$ such that $I^{\prime} \subset(\alpha, \inf I)$. Hence $\psi\left(t_{0}\right)=f\left(x_{0}\right)=y$ for some $t_{0} \in I^{\prime} \cap \mathscr{C}(\psi) \subset(\alpha, \beta) \cap \mathscr{C}(\psi)$.

- If $\inf I \in \bigcup_{n \in \mathbb{N}} F_{n}^{\prime}$, then $\inf I \in F_{n}^{\prime} \backslash F_{n-1}^{\prime}$ for some $n \in \mathbb{N}$. By (20),

$$
y \in\left[f\left(x_{0}\right), \psi(\inf I)\right]=\left[f\left(x_{0}\right), f\left(x_{0}\right)+\frac{1}{n}\right] \subset \psi[(\alpha, \inf I) \cap \mathscr{C}(\psi)] .
$$

So, there is a $t_{0} \in(\alpha, \inf I) \cap \mathscr{C}(\psi) \subset(\alpha, \beta) \cap \mathscr{C}(\psi)$ with $\psi\left(t_{0}\right)=y$.

Now assume that $\beta \in \bigcup_{n \in \mathbb{N}} F_{n}^{\prime} \backslash\{\sup I: I \in \mathcal{I}\}$. Then $\beta \in F_{n}^{\prime} \backslash F_{n-1}^{\prime}$ for some $n \in \mathbb{N}$. By (20),

$$
y \in\left[f\left(x_{0}\right), \psi(\beta)\right)=\left[f\left(x_{0}\right), f\left(x_{0}\right)+\frac{1}{n}\right) \subset \psi[(\alpha, \beta) \cap \mathscr{C}(\psi)] .
$$

Consequently, there is a $t_{0} \in(\alpha, \beta) \cap \mathscr{C}(\psi)$ with $\psi\left(t_{0}\right)=y$.

Case 2. If $y<f\left(x_{0}\right)$, then $\psi(\alpha)<f\left(x_{0}\right)$ and $\alpha \notin A_{1}$.

Then there is a $J \in \mathcal{J}$ such that $\alpha \in J$ and $\beta \notin \operatorname{cl} J$. Since, by (10), $\psi(\sup J)=$ $f\left(x_{0}\right)$ and $\psi\left\lceil\operatorname{cl} J=f\left\lceil\operatorname{cl} J \in \mathcal{S}_{s}\right.\right.$, there is a $t_{0} \in(\alpha, \sup J) \cap \mathscr{C}(\psi) \subset(\alpha, \beta) \cap \mathscr{C}(\psi)$ with $\psi\left(t_{0}\right)=y$. It follows that $\psi \in \mathcal{S}_{s}$.

Now define the function $g: \mathbb{R} \rightarrow \mathbb{R}$ as follows:

$$
g(x)= \begin{cases}\psi(x) & \text { if } x \in[c, d], \\ \psi(c) & \text { if } x \in(-\infty, c], \\ \psi(d) & \text { if } x \in[d, \infty) .\end{cases}
$$

Then clearly $g \in$ usc and by Lema 2.1 $g \in \mathcal{S}_{s}$. Finally we must show that $h=\max \{f, g\} \notin \dot{\mathcal{S}}_{s}$. Take $\alpha, \beta \in[c, d]$ such that $\alpha \in \bigcup \mathcal{J}, \beta \in \bigcup \mathcal{I}^{\prime}, \alpha<\beta$, and $h(\alpha)<f\left(x_{0}\right)<h(\beta)$. Obviously such numbers exist. It is easy to see that $\left[h=f\left(x_{0}\right)\right] \cap(\alpha, \beta) \subset \bigcup \mathcal{J} \cup A_{1}$. If $x \in J \cap\left[h=f\left(x_{0}\right)\right] \cap(\alpha, \beta)$ for some $J \in \mathcal{J}$, then since $f=g=h$ on $J$, using assumption (iii), we obtain that $x \notin \mathscr{C}(h)$. If $x \in A_{1} \cap(\alpha, \beta)$, then since $A_{1} \subset A$, by assumption (iv),

$$
\underline{\varliminf}\left(f, \bigcup \mathcal{J}, x^{+}\right)=\underline{\lim }\left(h, \bigcup \mathcal{J}, x^{+}\right)<f\left(x_{0}\right) \leq h(x)
$$


or

$$
\underline{\lim }\left(f, \bigcup \mathcal{J}, x^{-}\right)=\underline{\lim }\left(h, \bigcup \mathcal{J}, x^{-}\right)<f\left(x_{0}\right) \leq h(x),
$$

whence we also obtain that $x \notin \mathscr{C}(h)$. Consequently, $h(x) \neq f\left(x_{0}\right)$ for each $x \in(\alpha, \beta) \cap \mathscr{C}(h)$. So, $h=\max \{f, g\} \notin \mathcal{S}_{s}$, which completes the proof.

An immediate consequence of Theorem 2.5] is the following corollary.

Corollary 2.6. $\mathscr{C} \subset \mathcal{M}_{\max }\left(\dot{\mathcal{S}}_{\text {susc }}\right)$.

Pr o of. Suppose that $f \in \mathscr{C}$ and $f \notin \mathcal{M}_{\max }\left(\mathcal{S}_{\text {susc }}\right)$. Then $f \in \mathcal{S}_{\text {susc }}$ and there is a function $g \in \mathcal{S}_{\text {susc }}$ such that $\max \{f, g\} \notin \dot{\mathcal{S}}_{\text {susc }}$. Note that $\max \{f, g\} \in$ usc, whence $\max \{f, g\} \notin \mathcal{S}_{s}$. Using condition (iv) of Theorem 2.5 we directly obtain that $f \notin \mathscr{C}$, a contradiction.

Finally we will show that inclusions from Theorem 2.4 and Corollary 2.6 are proper.

Example 2.7. There is a function $f \in \mathcal{M}_{\text {max }}\left(\mathcal{S}_{\text {susc }}\right)$ which is not continuous.

Construction. Define the function $f: \mathbb{R} \rightarrow \mathbb{R}$ as follows:

$$
f(x)=\left\{\begin{array}{cl}
x & \text { if } x \in(-\infty, 0], \\
\sin \frac{1}{x}-1-x & \text { if } x \in(0, \infty) .
\end{array}\right.
$$

Clearly $f$ is upper semicontinuous but not continuous. Note that $\mathbb{R} \backslash \mathscr{C}(f)=\{0\}$. So, if $x \in \mathscr{C}(f)$, then condition (iv) of Theorem 2.5 is not fulfilled, and if $x=0$, then condition (ii) of Theorem 2.5 is not satisfied. Hence using Theorem 2.5 we obtain that $\max \{f, g\} \in \mathcal{S}_{s}$ for each function $g \in \mathcal{S}_{\text {susc }}$. Since the maximum of two upper semicontinuous functions is upper semicontinuous, we have $\max \{f, g\} \in \mathcal{S}_{\text {susc }}$. It proves that $f \in \mathcal{M}_{\max }\left(\mathcal{S}_{\text {susc }}\right)$.

Remark 2.8. There is an upper semicontinuous strong Świątkowski function $f$ such that $f \notin \mathcal{M}_{\max }\left(\mathcal{S}_{\text {susc }}\right)$.

Proof. By [7: Example 4.2] there are functions $f, g \in \dot{\mathcal{S}}_{\text {susc }}$ with $\max \{f, g\}$ $\notin \dot{\mathcal{S}}_{s}$, whence $f \notin \mathcal{M}_{\max }\left(\dot{\mathcal{S}}_{\text {susc }}\right)$.

\section{REFERENCES}

[1] FARKOVÁ, J.: About the naximum and the minimum of Darboux functions, Mat. Cas. Slov. Akad. Vied 21 (1971), No. 2, 110-116.

[2] GORDON, R. A.: The Integrals of Lebesgue, Denjoy, Perron, and Henstock. Grad. Stud. Math. 4, Amer. Math. Soc., Providence, RI, 1994.

[3] KUCNER, J.-PAWLAK, R. J.: On local characterization of the strong Światkowski property for a function $f:[a, b] \rightarrow \mathbb{R}$, Real Anal. Exchange 28 (2002/03), 563-572. 


\section{PAULINA SZCZUKA}

[4] MALISZEWSKI, A.: On the limits of strong Światkowski functions, Zeszyty Nauk. Politech. Łódz. Mat. 27 (1995), 87-93.

[5] SZCZUKA, P.: Maximal classes for the family of strong Światkowski functions, Real Anal. Exchange 28 (2002/03), 429-437.

[6] SZCZUKA, P.: Products of strong Światkowski functions, J. Appl. Anal. 12 (2006), $129-145$.

[7] SZCZUKA, P.: Maximums of upper semicontinuous strong Światkowski functions, Demonstratio Math. 44 (2011), 59-65.

Received 6. 10. 2011

Accepted 18. 6. 2012
Department of Mathematics Kazimierz Wielki University pl. Weyssenhoffa 11 PL-85-072 Bydgoszcz POLAND

E-mail: paulinaszczuka@wp.pl 